\title{
Relationship Between Postoperative Intraocular Lens Shift and Postoperative Refraction Change in Cataract Surgery Using Three Different Types of Intraocular Lenses
}

\author{
Takeshi Teshigawara (1) - Akira Meguro (1) - Nobuhisa Mizuki (1)
}

Received: June 24, 2021 / Accepted: August 12, 2021 / Published online: September 3, 2021

(c) The Author(s) 2021

\begin{abstract}
Introduction: Understanding the relationship between postoperative intraocular lens (IOL) shift and refractive change is crucial for the accuracy of predicted postoperative refraction (PPR). We assessed the relationships between different IOL fixation methods, haptic designs, and several metrics.

Methods: Single-center, open-label study which included 69 eyes. We preoperatively measured the anterior chamber depth (ACD), lens thickness (LT), axial length (AL), and PPR. AcrySof IQ $(n=27)$ and FineVision $(n=24)$ were fixed in the bag, and FEMTIS $(n=18)$ was fixed in capsulorhexis. At 1 day, 1 week, and 1 month postoperatively, we checked the IOL
\end{abstract}

Supplementary Information The online version contains supplementary material available at https:// doi.org/10.1007/s40123-021-00390-x.

T. Teshigawara $(\bowtie)$

Department of Ophthalmology, Yokosuka Chuoh Eye Clinic, 2-6 Odaki-cho, Yokosuka, Kanagawa 238-0008, Japan

e-mail: teshitake@gmail.com

T. Teshigawara

Tsurumi Chuoh Eye Clinic, 1-2-1 Tsurumi-chuo,

Tsurumi, Yokohama, Kanagawa 230-0051, Japan

T. Teshigawara · A. Meguro · N. Mizuki

Department of Ophthalmology, Yokohama City

University School of Medicine, 3-9 Fukuura,

Kanazawa, Yokohama, Kanagawa 236-0004, Japan position and refraction and compared the IOL shift, refraction change, and the margin of error of the predicted PPR (PR-PPR difference). We also analyzed the correlation between postoperative and preoperative variables.

Results: FEMTIS showed the highest stability in terms of IOL shift and refraction. The in-thebag-fixated IOLs showed a significant forward shift between 1 day and 1 week postoperatively. There were significant differences in the PR-PPR difference between the IOLs. ACD and AL showed significant positive correlations, and LT showed a significant negative correlation with IOL shift and change in PR between 1 day and 1 week, but not between 1 week and 1 month postoperatively.

Conclusion: The relationship between postoperative IOL shift and refraction change varied according to IOLs with different haptic types. Capsulorhexis fixation may be more stable than in-the-bag fixation. Furthermore, preoperative ACD, LT, and AL may be useful factors to predict IOL shift, change in PR, and to a lesser extent the degree of the margin of error in clinical practice. Our findings may improve the accuracy of PPR and refractive outcomes.

Keywords: Capsulorhexis; IOL in the Bag; IOL Shift; Optic Captured Plate-Haptics; Postoperative Refraction 


\section{Key Summary Points}

Why carry out this study?

Understanding the nature of postoperative intraocular lens (IOL) shift, refractive change, and margin of error in predicted postoperative refraction (PPR) is crucial for the accuracy of PPR.

We aimed to assess the relationships between different fixation methods of IOLs with different haptic designs and describe the correlation between IOL shift, postoperative refraction, margin of error in PPR, and preoperative biometric variables.

\section{What was learned from the study?}

The relationship between the postoperative IOL shift and refraction change varied among IOLs with different haptic designs; preoperative anterior chamber depth, lens thickness, and axial length may have predictive roles.

The study results indicate that capsulorhexis may be more stable than inthe-bag IOL fixation, leading to improved accuracy of PPR and better refractive outcomes.

\section{INTRODUCTION}

Cataract surgery has become a routine part of refractive surgery. One of the main causes of postoperative patient dissatisfaction is residual refractive error [1]. An ultrasonography-based A-scan was traditionally used to measure axial length $(\mathrm{AL})$, which is the most common cause of error in intraocular lens (IOL) calculations [2]. The use of partial coherence interferometry improved the accuracy of AL measurements to five times that of the A-scan [3]. Additionally, new IOL calculation formulae have increased the accuracy of predicted postoperative refraction (PPR) [4]. However, postoperative refraction changes are sometimes observed, even in the early postoperative period. This makes choosing the IOL power of the fellow eye challenging.

Previous studies have reported a relationship between postoperative IOL shift and postoperative refraction. Nejima et al. indicated that postoperative IOL shift could induce postoperative refractive changes [5]. Erickson demonstrated the influence of the postoperative IOL position on the postoperative refractive error [6]. The results of these studies suggest that the stability of IOLs may be an important factor in improving the accuracy of PPR. Remón et al. investigated the influence of material and haptic design on the mechanical stability of IOLs and concluded that haptic design had more influence on stability than material properties [7]. Regarding IOL placement methods, in-thebag fixation is the most common procedure, while capsulorhexis has been recently introduced $[8,9,10]$.

Given that postoperative refraction in the operated eye is often considered when choosing the IOL power of the fellow eye, knowledge of the postoperative IOL shift and its influence on postoperative refraction changes in the early postoperative period is crucial for improving the accuracy of PPR.

The objective of this study was to investigate the postoperative IOL shift in three different IOLs with different haptic designs and different IOL fixation methods, as to the best of our knowledge no previous study has done so. In addition, we aimed to measure their effect on postoperative refraction changes in the early postoperative period. The correlations between IOL shift, postoperative refraction changes, and preoperative biometric variables were also analyzed.

\section{METHODS}

This single-center, open-label study was conducted at the Yokosuka Chuo Eye Clinic, and operations were performed between May 2018 and November 2019. Detailed information about this research was provided and informed 
consent was obtained from all participants. All research procedures were performed in accordance with the Declaration of Helsinki. This study was approved by the Ethics Committee of the Yokosuka Chuo Eye Clinic.

\section{Patient and IOL Selection}

The study included 69 eyes of 69 patients. The AcrySof IQ (model SN60WF; Alcon, Fort Worth, TX, USA), FineVision Pod F (PhysIOL, Liège, Belgium), and FEMTIS (Oculentis, Berlin, Germany) lenses were used in 27, 24, and 18 eyes, respectively. All operations were performed by the same surgeon at the Yokosuka Chuo Eye Clinic. All participants had age-related cataracts with a nucleus grading of $>2$ based on the Emery-Little classification and were randomly chosen by an ophthalmologist. Patients with pseudoexfoliation glaucoma, uveitis, proliferative diabetic retinopathy, or a history of intraocular surgery, laser treatment, and retinal problems, or subjects with corrected postoperative visual acuity 20/40, were excluded.

The eye specialist informed all patients about the characteristics of each IOL. The IOLs were selected according to patient demands. FEMTIS is a haptic-designed, hydrophilic, acrylic plate with four flanges for capsulorhexis fixation (Fig. 1a). It has an anteroposterior, aspheric, biconvex optic with a diameter of $5.7 \mathrm{~mm}$, an overall length of $10.5 \mathrm{~mm}$, and no haptic angulation $\left(0^{\circ}\right)$. FineVision is a hydrophobic, acrylic, single-piece IOL with double C-loop haptics (Fig. 1b). It has an anteroposterior, aspheric, biconvex optic with a diameter of $6.0 \mathrm{~mm}$, an overall length of $11.4 \mathrm{~mm}$, and a haptic angulation of $5^{\circ}$. AcrySof IQ is a hydrophobic, acrylic, single-piece IOL with a single C-loop haptic (Fig. 1c). It has an anteroposterior, aspheric. biconvex optic with a diameter of $6.0 \mathrm{~mm}$, an overall length of $13.0 \mathrm{~mm}$, and a haptic angulation of $0^{\circ}$.

\section{Pre- and Postoperative Examinations}

All patients underwent complete preoperative ophthalmic examinations. Nucleus grading was classified according to slit-lamp examination findings by the same eye specialist. The vision test for subjective refraction was performed by the same technician for all patients. Sweptsource anterior segment optical coherence tomography imaging was performed using the a

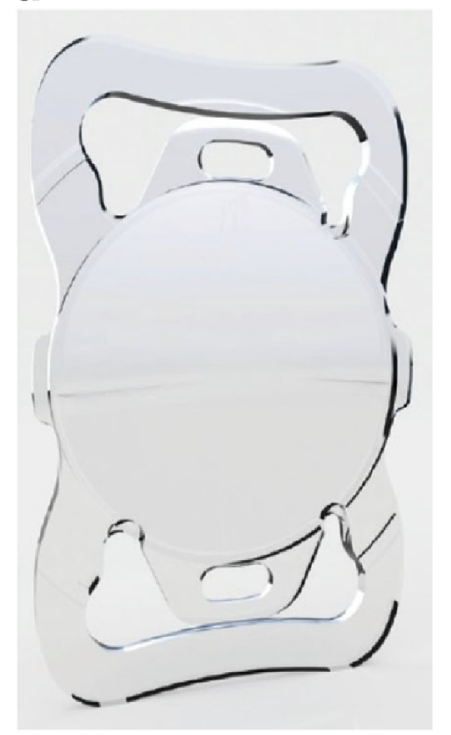

b

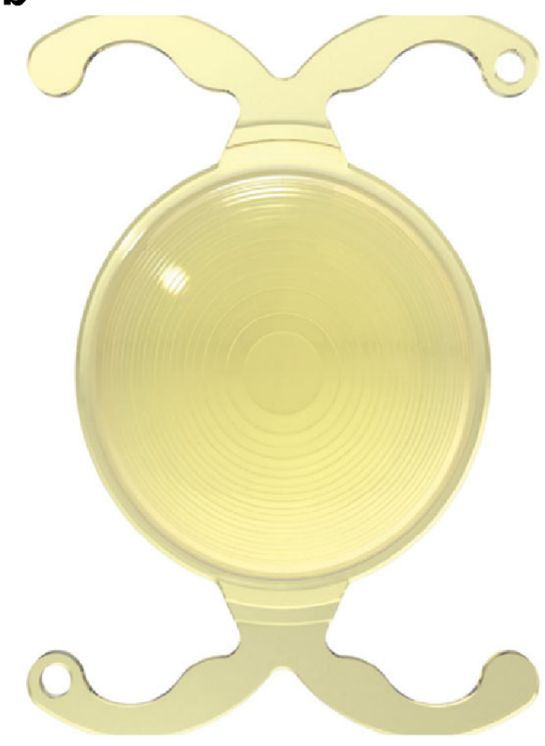

C

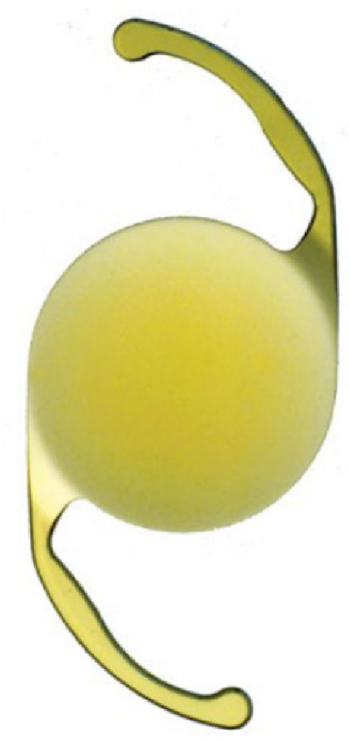

Fig. 1 Types of IOLs: AcrySof IQ (a), FineVision (b), FEMTIS (c) 


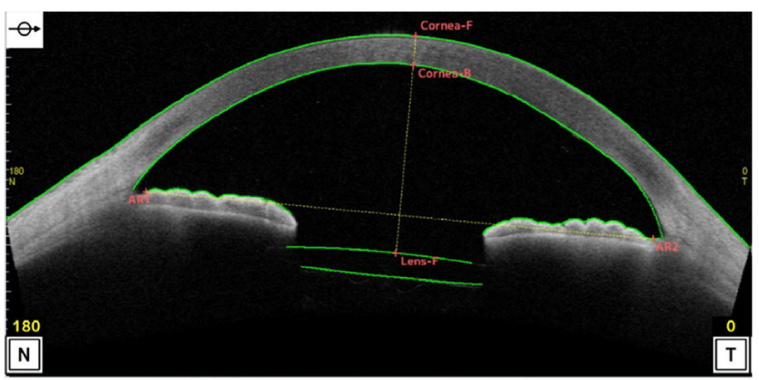

Fig. 2 Measurement of the postoperative IOL position (corneal $\mathrm{F}$ to lens $\mathrm{F}$ )

CASIA2 system (Tomey Corp., Nagoya, Japan) to detect anatomical abnormalities. Anterior chamber depth (ACD), lens thickness (LT), and $\mathrm{AL}$ were measured using an IOLMaster 700 device (Carl Zeiss, Oberkochen, Germany). The PPR and recommended IOL power were calculated using the IOLMaster 700.

In addition to the ophthalmic examinations, the IOL position was measured using CASIA2 on postoperative day 1 , week 1 , and month 1 . The lens position was measured from the apex of the corneal surface to the center of the IOL (Fig. 2). Subjective postoperative refraction was evaluated by the same technician. Refraction was reported as the spherical equivalent (SE). Due to a possible margin of error caused by different examiners, vision and postoperative IOL positions were measured by the same experienced examiner.

\section{Surgical Technique}

All operations involved femtosecond laserassisted cataract surgery using the LenSx platform (Alcon Laboratories, Inc., Fort Worth, TX, USA). The same surgeon performed the laser and manual operations. Before the laser process, topical anesthesia with $0.4 \%$ oxybuprocaine eye drops (Benoxyl; Santen Pharmaceutical Co., Osaka, Japan) was administered after maximal dilation of the pupil with $0.5 \%$ tropicamide and $0.5 \%$ phenylephrine hydrochloride (Mydrin-P, Santen Pharmaceutical, Osaka, Japan). Preoperative planning of capsulotomy and lens fragmentation was performed using the LenSx platform. A $5.0 \mathrm{~mm}$ capsulotomy and chop- and-cylinder fragmentation pattern was selected, with three chops, each measuring $6 \mathrm{~mm}$ in length. The posterior offset was set at $800 \mu \mathrm{m}$. No dock-related issues were observed while using SoftFit ${ }^{\mathrm{TM}}$ (Alcon Laboratories, Inc., Fort Worth, TX, USA) in all cases. A $5.0 \mathrm{~mm}$ capsulotomy centered on white-to-white was created with $8.0 \mathrm{~mJ}$ of energy (spot and layer separations: $9 \mu \mathrm{m}$ each). Nuclear fragmentation was performed using the chop-and-cylinder technique with $8.0 \mathrm{~mJ}$ of energy (spot and layer separations: $9 \mu \mathrm{m}$ each). The anterior and posterior delta values were $350 \mu \mathrm{m}$.

A temporal clear corneal incision of $2.40 \mathrm{~mm}$ was made using an ophthalmic knife (MANI Ophthalmics, Tochigi, Japan). The anterior chamber was filled with a dispersive viscoelastic VISCOAT (Alcon Laboratories, Fort Worth, TX, USA) and a cohesive viscoelastic OPELEAD (Senju Pharmaceutical Co., Ltd., Osaka, Japan) using the soft-shell technique. Capsulotomy was carefully performed using capsule forceps, and cortico-cleaving hydrodissection was performed. Emulsification and aspiration of the fragmented lens and aspiration of the cortex were conducted using the Centurion ${ }^{\circledR}$ Vision System (Alcon Laboratories, Inc., Fort Worth, TX, USA). After filling the capsule bag with OPELEAD, the IOLs were inserted into the bag using an IOL injector. The attachment of the enclaved flanges to the rim of the capsulorhexis was performed using the reverse optic capture technique. The viscoelastic material was removed before watertight corneal wound closure was confirmed.

\section{Data Analysis}

The postoperative IOL longitudinal shift was calculated as follows: the IOL shift between 1 day and 1 week was the IOL position on postoperative day 1 minus the position at postoperative week 1 ; the IOL shift between 1 week and 1 month was defined as the IOL position on postoperative week 1 minus the position on postoperative month 1 . A positive IOL shift indicates that the IOL showed a forward shift, whereas a negative number indicates a backward shift during a particular period. 
The postoperative refraction change was calculated as follows: the refraction change between 1 day and 1 week was the PR in postoperative week 1 minus the PR on postoperative day 1 ; the refraction change between 1 week and 1 month was defined as the PR in postoperative month 1 IOL minus the PR in postoperative week 1 . These were analyzed in terms of both the real and absolute values. In real values, a positive number refraction change indicates a hyperopic shift, whereas a negative number indicates a myopic shift during a particular period. In absolute values, the numbers indicate the degree of refraction change during a particular period.

The margin of error of postoperative refraction, which was calculated as the postoperative refraction minus the predicted postoperative refraction (PR-PPR) in three different types of IOLs, was also analyzed at 1 day, 1 week, and 1 month after surgery. These results are shown as real and absolute values. When analyzing real values, a positive PR-PPR value indicates that the postoperative refraction (PR) showed a hyperopic shift compared to the PPR, whereas a negative PR-PPR value indicated that the postoperative refraction showed a myopic shift compared to the PPR.

We performed an analysis of variance (ANOVA) test for the numerical values that followed a normal distribution (assessed with the Shapiro-Wilk test), the Kruskal-Wallis test for numerical values not following a normal distribution, and Fisher's exact test to analyze data from categorical variables. We tested the following numerical variables: IOL shift between 1 day and 1 week and 1 week and 1 month postoperatively, and the PR-PPR at 1 day, 1 week, and 1 month postoperatively. We investigated whether there were statistically significant differences in terms of eye laterality (right/left), sex, age, preoperative ACD, LT, and AL; IOL shift and change in PR between 1 day and 1 week and 1 week and 1 month among the types of IOLs; and PR-PPR difference at 1 day, 1 week, and 1 month postoperatively among the categories (types) of IOLs.

Furthermore, we used Dunn's test [11] to perform multiple comparisons with a Bonferroni adjustment of the $p$-value. We determined which categories (types) of IOLs had significant differences in IOL shifts and changes in PR between 1 day and 1 week and 1 week and 1 month. Moreover, we determined the PR-PPR differences at 1 day, 1 week, and 1 month postoperatively.

Spearman's rank correlation coefficients were used to examine whether preoperative $A C D$, preoperative $\mathrm{LT}$, and preoperative $\mathrm{AL}$ correlated with the following variables during each study period: postoperative IOL shifts, the degree of change in PR, and the degree of margin of error. The above tests were only performed for two types of IOLs (FineVision and AcrySof IQ) to ensure consistency in the types of lenses being compared (i.e., in-the-bag-fixation IOLs) and also because of the small number of patients in the capsulorhexis (FEMTIS) group.

The significance level was set at $p<0.05$. All statistical analyses were performed using R (R Foundation for Statistical Computing, Vienna, Austria; https://www.R-project.org/).

\section{RESULTS}

The descriptive statistics and the results of the ANOVA, Kruskal-Wallis test, and Fisher's exact test, according to the three IOL types, are presented in Table S1. Biometric measurements are presented in Table 1 . The proportion of male patients in the AcrySof IQ, FineVision, and FEMTIS lens groups was 35\%, 42\%, and 43\%, respectively.

Eye laterality (right/left) and sex were not significantly different $(p>0.05)$ among the different IOL groups. Age was significantly different $\left(x^{2}=6.2706, p=0.043\right)$ between the IOL groups, which was not confirmed by Dunn's test with the Bonferroni adjustment (all $p$ values $>0.05$ )

The results of the ANOVA for preoperative ACD and LT and the Kruskal-Wallis test for AL also showed that they were not significantly different $(p>0.05)$ among the different types of IOLs.

The IOL shift from 1 day to 1 week postoperatively, tested in both real and absolute values, showed significant differences (for both cases: $p<0.01$ ) among the IOL types (Fig. 3a, b, 
Table 1 Clinical characteristic of the eyes in each intraocular lens group

\begin{tabular}{llllll}
\hline Lens type & $\boldsymbol{n}$ & Median & Confidence level & Lower percentile & Upper percentile \\
\hline Age & & & & & \\
1. FEMTIS & 18 & 68 & 0.95 & 59 & 72.5 \\
2. FineVision & 24 & 73 & 0.95 & 70 & 76 \\
3. AcrySof IQ & 27 & 75 & 0.95 & 69 & 77 \\
ACD (mm) & & & & & 3.02 \\
1. FEMTIS & 18 & 2.85 & 0.95 & 2.26 & 3.06 \\
2. FineVision & 24 & 2.77 & 0.95 & 2.47 & 2.92 \\
3. AcrySof IQ & 27 & 2.76 & 0.95 & 2.52 & 4.72 \\
LT (mm) & & & & 4.45 & 4.7 \\
1. FEMTIS & 18 & 4.53 & 0.95 & 4.25 & 4.72 \\
2. FineVision & 24 & 4.61 & 0.95 & 4.43 & 24.4 \\
3. AcrySof IQ & 27 & 4.62 & 0.95 & & 24.9 \\
AL (mm) & & & & 22.9 & 24.3 \\
1. FEMTIS & 18 & 23.7 & 0.95 & 23.2 & 23.1 \\
2. FineVision & 24 & 23.6 & 0.95 & 0.95 & \\
3. AcrySof IQ & 27 & 23.4 & &
\end{tabular}

$A C D$ anterior chamber depth, $L T$ lens thickness, $A L$ axial length

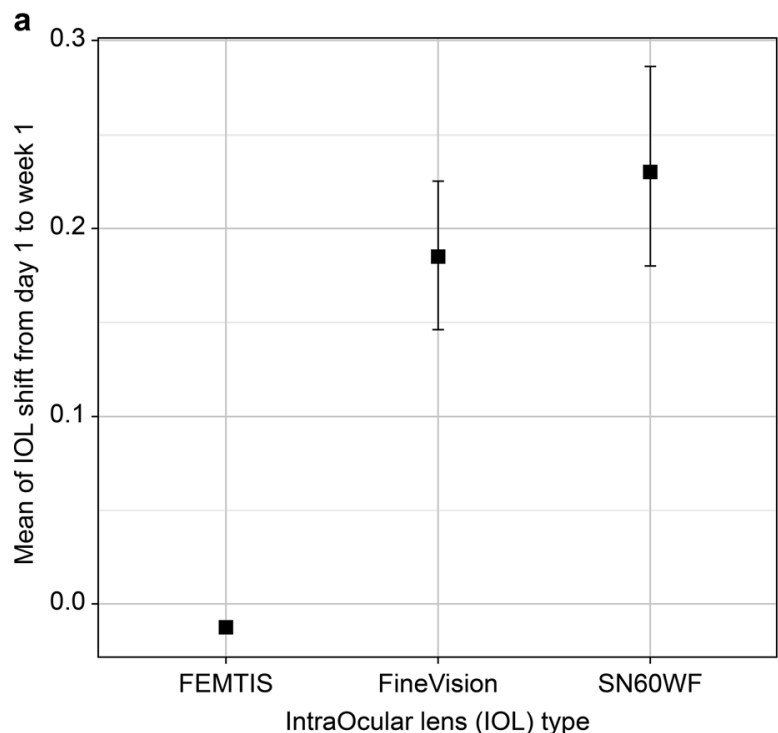

Fig. 3 a, b Graph showing mean lens shift (in $\mathrm{mm}$ ) in real (a) and absolute (b) values between 1 day and 1 week postoperatively by lens type. Square dots represent the

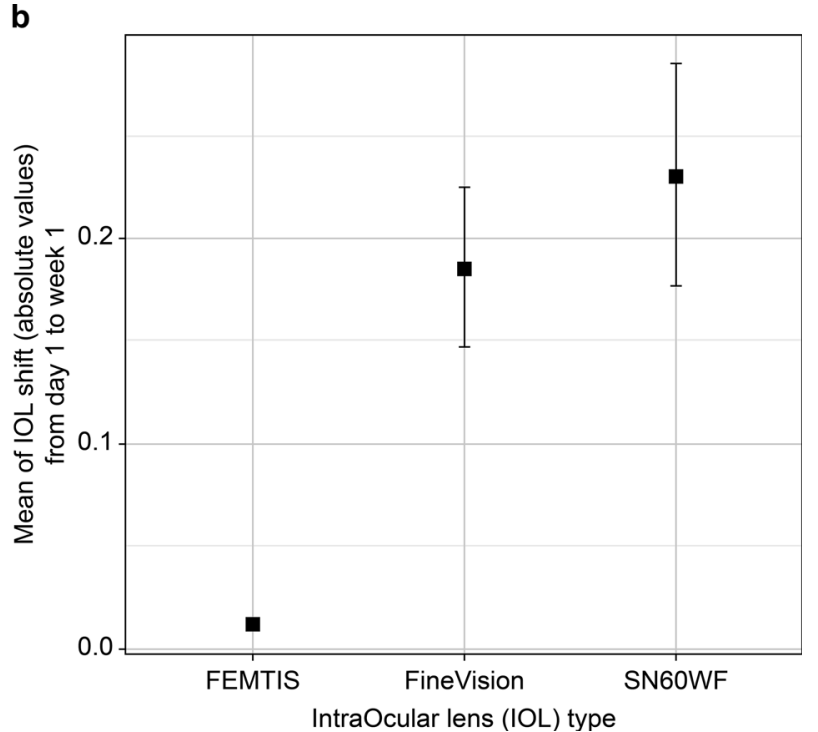

median values of the shifts, and the upper and lower whiskers represent the upper and lower percentiles 


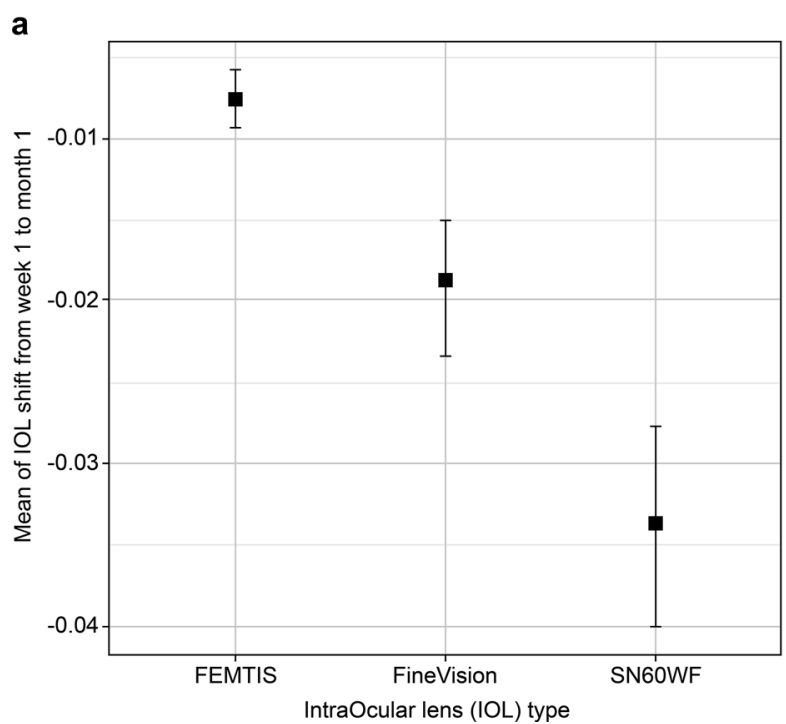

Fig. 4 Graph showing mean lens shift (in $\mathrm{mm}$ ) in real (a) and absolute (b) values between 1 week and 1 month postoperatively by lens type. Square dots represent the

a

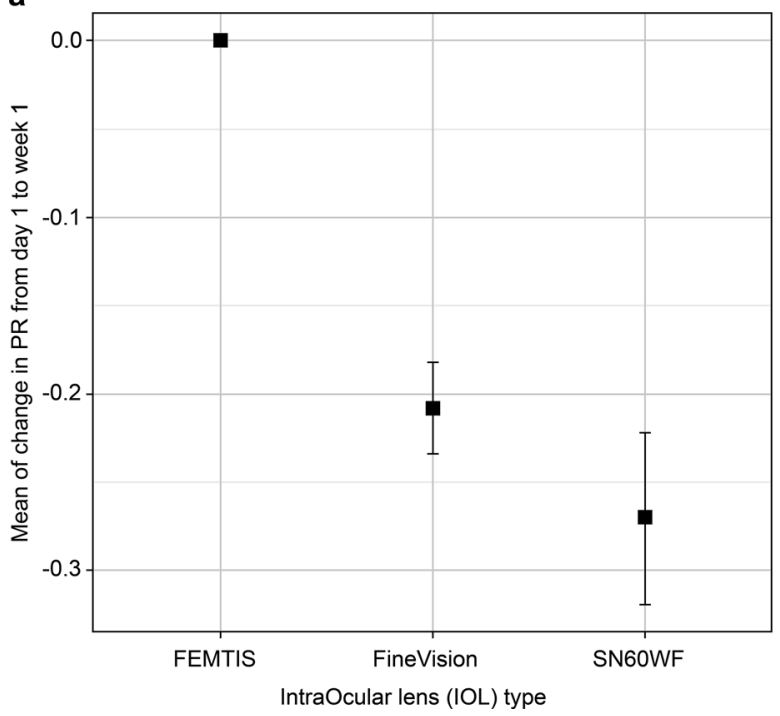

Fig. 5 Graph showing the mean change in PR in real (a) and absolute (b) values between day 1 and week 1 postoperatively by lens type. Square dots represent the

respectively). The IOL shift was significantly lower with FEMTIS than with both FineVision and AcrySof IQ $(Z=-5.199565, p<0.01$; and $Z=-5.9472881, p<0.01$, respectively). The IOL shift in real values was negative for the b

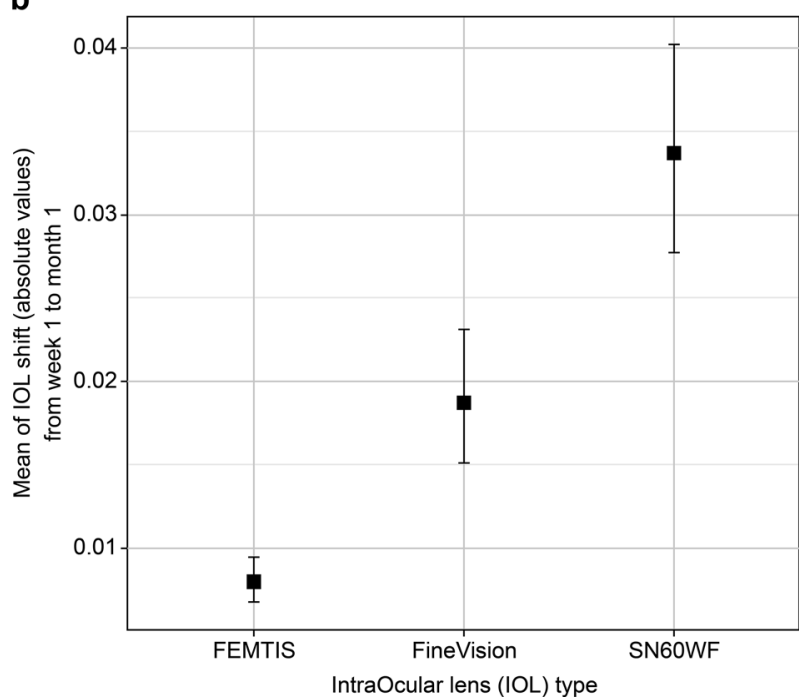

median values of the shifts, and the upper and lower whiskers represent the upper and lower percentiles

b

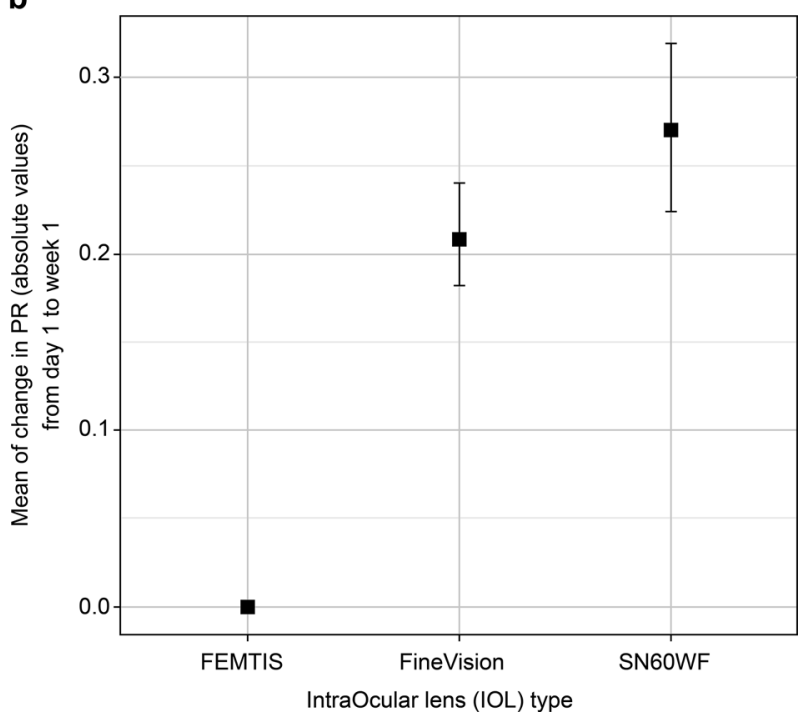

median values of the shifts, and the upper and lower whiskers represent the upper and lower percentiles

FEMTIS and positive for both FineVision and SN60WF (Fig. 3a).

The IOL shift from 1 week to 1 month postoperatively, tested in both real and absolute values, showed significant differences $(p<0.01)$ among the IOL types (Fig. 4a, b). Dunn's test 
a

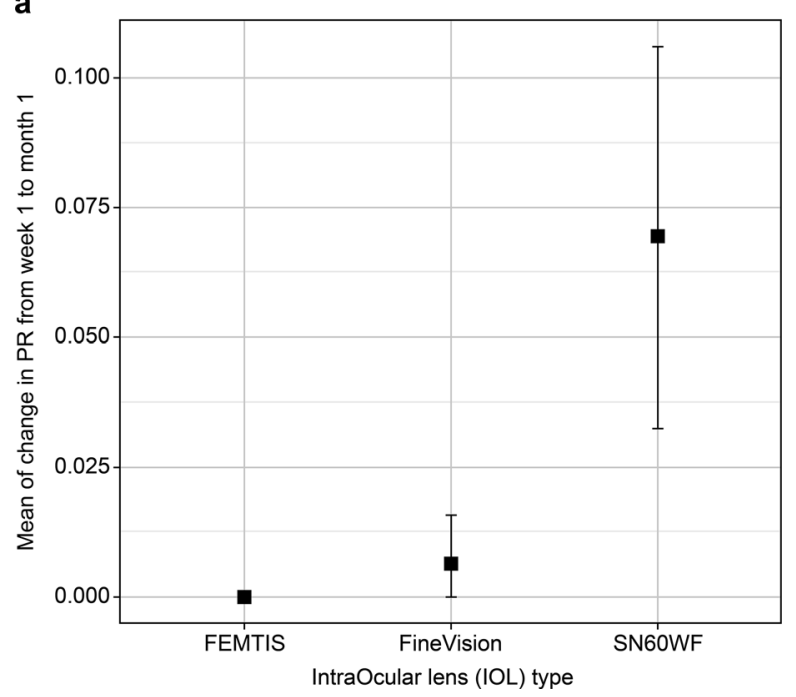

Fig. 6 Graph showing the mean change in PR in real (a) and absolute (b) values between week 1 and month 1 postoperatively by lens type. Square dots represent the

showed that the IOL shift from 1 week to 1 month postoperatively was significantly smaller with FEMTIS than with FineVision and AcrySof IQ $(Z=3.474281, p<0.01 ; \quad$ and $Z=6.099305, p<0.01$, respectively). In addition, the IOL shift showed significant differences $(Z=2.754178, p<0.05)$ between the FineVision and AcrySof IQ IOL types. The IOL shift in real values was negative for all three FEMTIS, FineVision, and AcrySof IQ IOLs (Fig. 4a).

The overall mean postoperative refraction (PR) was $-0.090 \pm 0.153$. The change between 1 day and 1 week, tested in both real and absolute values, showed significant differences $(p<0.01)$ among the IOL types (Fig. 5a, b), with the FEMTIS IOL showing smaller changes than both the FineVision and AcrySof IQ IOLs. The PR change between 1 week and 1 month, tested in both real and absolute values, showed significant differences $(p<0.01)$ between the IOL types (Fig. 6a, b), with the FEMTIS IOL showing smaller changes than both the FineVision and AcrySof IQ IOLs.

The PR-PPR difference was $-0.059 \pm 0.063$. On day 1 , tested in absolute values, significant differences $(p<0.01)$ were found among the IOL types (Fig. 7a), with the AcrySof IQ IOL b

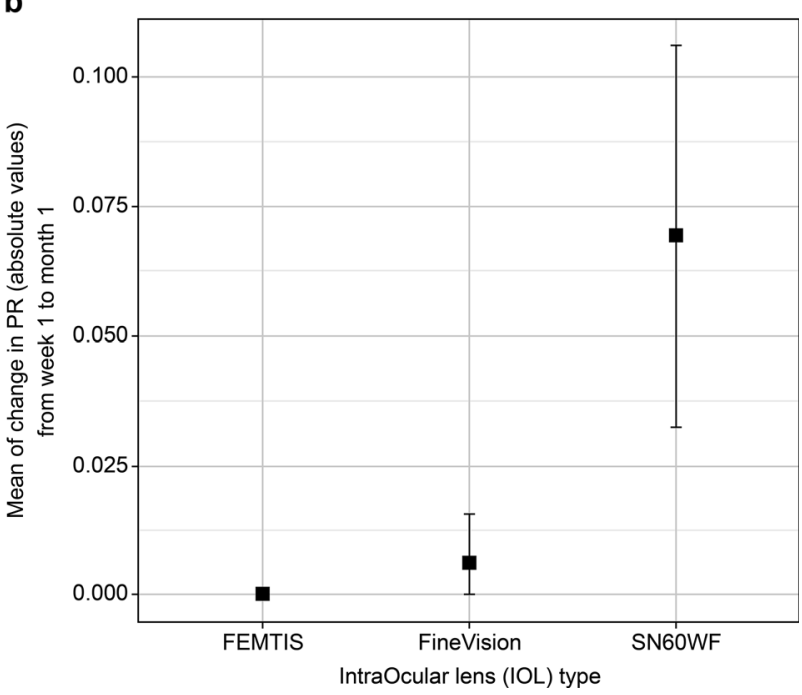

median values of the shifts, and the upper and lower whiskers represent the upper and lower percentiles

having the highest absolute values at this time point. Dunn's test showed that the absolute values for the PR-PPR differences at 1 day after the operation were not significantly different $(Z=-1.24, p>0.05)$ between the FEMTIS and FineVision lenses, while they were significantly different between the FEMTIS and AcrySof IQ lenses $(Z=-4.53, p<0.01)$ and the FineVision and AcrySof IQ lenses $(Z=-3.54, p<0.01$; Table S2). The PR-PPR difference at 1 week, tested in absolute values, showed significant differences $(p<0.05)$ among the IOL types (Fig. 7b). The absolute value of the PR-PPR difference at 1 week after the operation was significantly different $(Z=-3.38, \quad p<0.01)$ between the FEMTIS and FineVision lenses, while it was not significantly different between the FEMTIS and AcrySof IQ lenses $(p>0.05)$ or the FineVision and AcrySof IQ lenses $(p>0.05)$. The PR-PPR difference at 1 month, tested in absolute values, showed significant differences $(p<0.05)$ among the IOL types (Fig. 7c). Dunn's test showed that the absolute value of the PR-PPR difference at 1 month after the operation was significantly different $(Z=-3.33, p<0.01)$ between the FEMTIS and FineVision lenses, while it was not significantly different between the FEMTIS and AcrySof IQ 
a

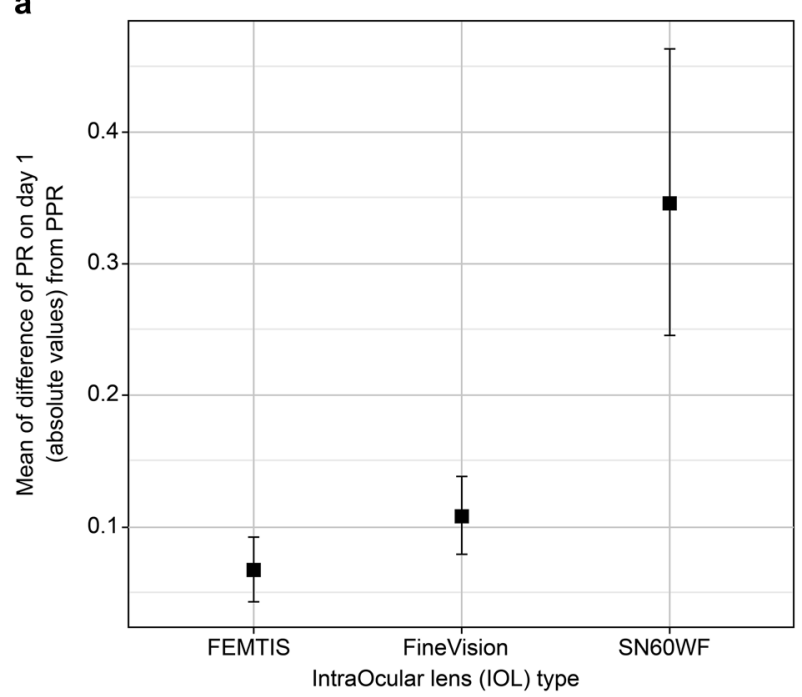

C

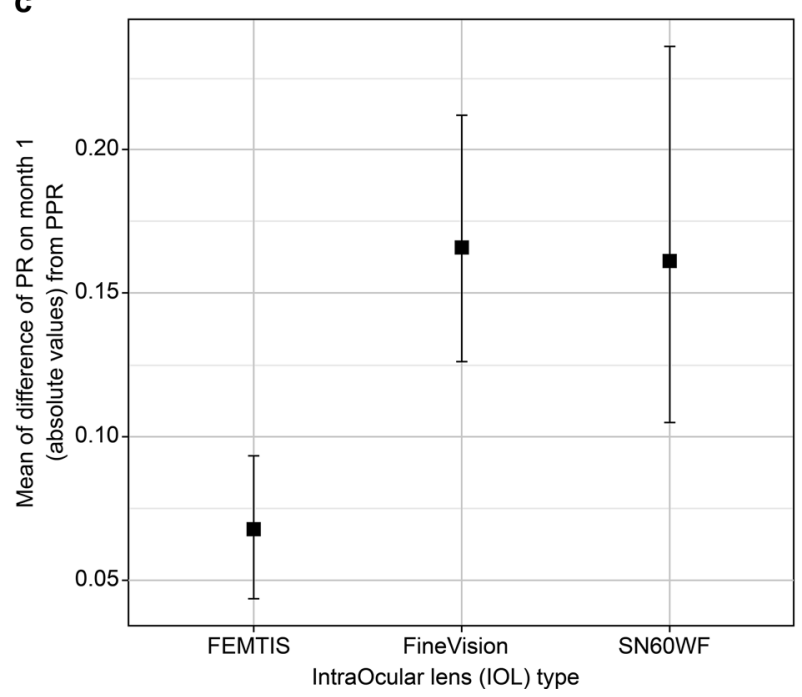

b

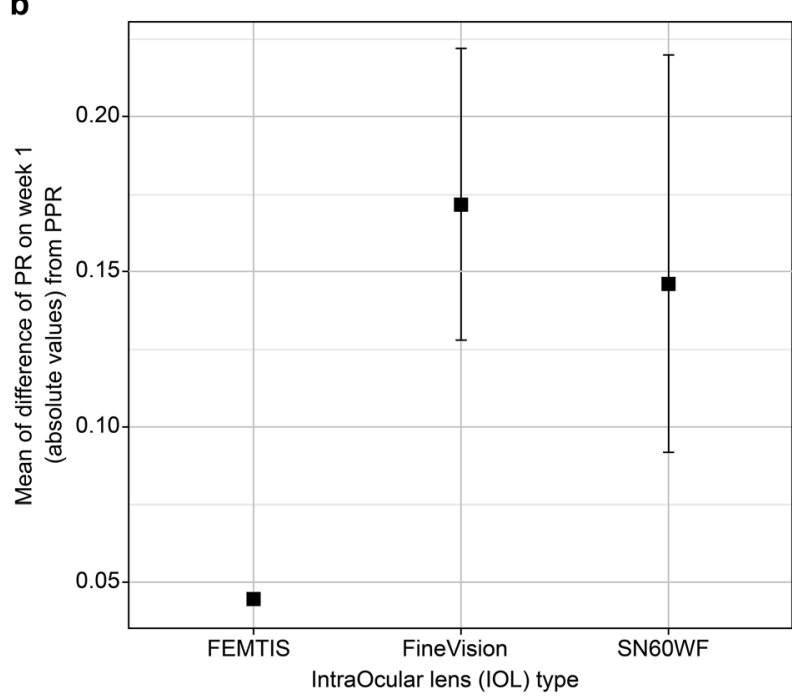

Fig. 7 Graph showing PR-PPR difference at 1 day (a), 1 week (b), and 1 month (c) postoperatively by lens type. Square dots represent the median values of the shifts, and the upper and lower whiskers represent the upper and lower percentiles

lenses $(p>0.05)$ or the FineVision and AcrySof IQ lenses $(p>0.05)$.

Spearman's rank correlation coefficient tests for the two in-the-bag IOLs (FineVision and AcrySof IQ) are shown in Tables S3-S5. The IOL shift from 1 day to 1 week showed a strong positive correlation with both ACD and AL $($ rho $=0.83$, rho $=0.90$, respectively; $p<0.01)$, and a strong negative correlation with LT $($ rho $=-0.90 ; p<0.01)$. The changes in PR (absolute values) from 1 day to 1 week showed a strong positive correlation with both ACD and $\mathrm{AL} \quad(\mathrm{rho}=0.71, \quad$ rho $=0.77, \quad$ respectively; $p<0.01$ ), and a strong negative correlation with LT (rho $=-0.77 ; p<0.01$ ).

The PR-PPR difference on day 1 in absolute values showed a weak positive correlation with both ACD and AL (rho $=0.44$, rho $=0.36$, respectively; $p<0.05$ ), and a weak negative correlation with LT (rho $=-0.36 ; p<0.05)$. The 
PR-PPR difference at week 1 in absolute values did not show any significant correlation with $\mathrm{ACD}, \mathrm{AL}$, or LT $(p>0.05)$. The PR-PPR difference at month 1 in absolute values did not show any significant correlation with ACD $(p>0.05)$, but it did show a weak positive correlation with $\mathrm{AL}$ (rho $=0.28, p<0.05$ ) and a weak negative correlation with LT (rho $=-0.31 ; p<0.05)$.

\section{DISCUSSION}

Outcomes following IOL implantation have become an important research area [12, 13]. Based on our clinical experience, we wondered why some patients showed refraction changes after surgery and why these changes varied from patient to patient and by IOL type. Erickson insisted that postoperative longitudinal shifts might be the principal contributors to postoperative refractive errors [6]. Considering that one of the main causes of patient dissatisfaction after IOL implantation is postoperative refractive errors [1], ophthalmologists need to understand postoperative IOL longitudinal shifts and their influence on postoperative refractive changes [14]. This knowledge can also help clinicians better predict postoperative refraction in the early postoperative period and choose a suitable IOL power for the fellow eye [14]. Furthermore, understanding the relationship between preoperative biometric variables, postoperative IOL shifts, and postoperative refraction changes is also useful for improving the accuracy of PPR.

In this study, the FEMTIS IOL, which was fixed to the anterior capsule, showed the least postoperative IOL longitudinal shift, the least change in postoperative refraction, and the smallest difference between PPR and actual postoperative refraction in the early postoperative period. These results are in line with previous studies that also used a capsulorhexisfixated IOL with slightly different designs and indicated greater stability with FEMTIS. Ganesh et al. and Dick et al. reported similar results in terms of postoperative IOL longitudinal shift and postoperative refraction changes $[8,9]$. Kim et al. investigated lens stability and refractive outcomes after cataract surgery using primarily posterior capsulorhexis fixation and concluded that this fixation method was useful in stabilizing the postoperative IOL position and minimizing postoperative refractive changes [15].

The success of the capsule fixation technique requires a consistent size and shape of the capsulorhexis [16]. However, ensuring a consistent size and shape of the capsulorhexis is challenging even for an experienced surgeon. Recently released cutting-edge devices, such as femtosecond laser systems, selective capsulotomy laser systems, and precision pulse capsulotomy, can be used to tackle these challenges [17-21]. Among these, the femtosecond laser system is the only device that is used to create the capsulorhexis on the visual axis. One previous study reported that aspheric IOLs can be very sensitive in quality of vision due to decentration depending on the aberration correction concept of the IOL design. In aberration-correcting IOLs, coma aberration and astigmatism significantly increase with increasing decentration [22]. Kránitz et al. showed that IOL decentration was six times as likely to occur when the capsulorhexis was created manually as when it was created using the femtosecond laser technique [20]. Some studies have reported that IOL decentration can also induce postoperative astigmatism [6]. The results of these studies imply that a combination of anterior capsule fixation of the IOL and capsulorhexis creation with a femtosecond laser may be ideal for stabilizing the postoperative IOL position and postoperative quality of vision.

Several studies have investigated postoperative refractive changes and postoperative IOL longitudinal shifts in in-the-bag fixated IOLs [5, 23-25]. In our study, the capsulorhexis-fixation IOL FEMTIS was the most stable throughout the study period. In contrast, in-the-bagfixation IOLs AcrySof IQ and FineVision showed significant forward shifts between 1 day and 1 week, which means that these IOLs showed myopic shifts during this period. The degree of the shift between 1 day and 1 week was the most significant compared to the other periods. One of the main differences between previous research and our work are the periods of the assessments. While most previous studies assessed shifts of in-the-bag-fixation IOLs 
during a relatively long postoperative period, we compared them in the early postoperative period, up to 1 month after surgery. Similar to our study, Koeppl et al. [26] investigated postoperative IOL longitudinal shifts and postoperative refraction changes among in-the-bagfixation IOLs in the early postoperative period; they reported that the changes in both were the most remarkable between 1 day and 1 week after the operation, after which they became more stable, which corresponds to our findings. Both their and our results indicate that IOL longitudinal shifts and refraction changes may be observed even in the early postoperative period. Therefore, it may be wise to consider these tendencies when choosing the IOL power of the fellow eye.

In our study, AcrySof IQ and FineVision showed similar overall effects on postoperative IOL longitudinal shift, but the magnitudes of the effects were different. These two in-the-bagfixation IOLs have different specifications, including the material they are made of, their length, edge designs, and haptic designs, all of which can cause differences in the magnitude of the postoperative shift. In fact, previously published studies reported that capsular bag shrinkage, IOL design (haptic or optic), and IOL material may influence changes in postoperative longitudinal IOL shifts. Koeppl et al. insisted that significant shrinkage of the capsular bag in the first postoperative week was correlated with a forward IOL shift [26]. Petternel et al. assessed the influence of optic-edge design and optic-haptic angulation of IOLs on postoperative longitudinal shifts [27] and found that the influence of optic-haptic angulation was significantly greater than that of edge design. Wirtitsch et al. investigated the effect of haptic design on postoperative IOL longitudinal shifts and reported that single-piece IOLs were more stable than three-piece IOLs [14]. It has also been reported that the IOL material influences postoperative IOL shifts [23]. Thus, the various specifications and characteristics of different IOLs may cause postoperative IOL shifts. Therefore, it is difficult to conclude which IOL specification and characteristics primarily resulted in the differences in magnitudes of the postoperative IOL shifts in both acrylic single- piece IOLs with different haptics. These issues will be addressed in future studies.

Our results also indicated significant differences in the margin of error of predicted postoperative refraction (PR-PPR) between in-thebag-fixation IOLs and capsulorhexis-fixation IOLs. Although these differences were not consistent over time, the capsulorhexis-fixation IOL FEMTIS showed a smaller margin of error than the in-the-bag-fixation IOLs throughout the study period. In particular, the PR-PPR with FEMTIS was significantly smaller than that of the in-the-bag-fixation IOLs at 1 day postoperatively.

As a result, FEMTIS was most stable in all aspects in this study including postoperative IOL shift, change in postoperative refraction (PR), and PR-PPR. Taken together, these results suggest that optic capture in capsulorhexis fixation may be more stable in terms of the postoperative IOL position and possibly refractive outcomes than in-the-bag fixation. Therefore, capsulorhexis fixation may be associated with improved PPR accuracy. This notion is supported by previous research on the mechanism of postoperative movement of in-the-bag fixated IOLs. They reported that postoperative IOL decentration and dislocation can be attributed to a combination of the high flexibility of inthe-bag-fixation IOLs, their haptic configuration, and the postoperative fibrosis and shrinkage of the capsular bag [28-30]. Therefore, we can theoretically assume that since the optics of the capsule-fixated IOLs are fixed in the capsulorhexis, they may be less influenced by postoperative fibrosis and shrinkage of the bag.

Interestingly, when comparing the two inthe-bag IOLs with each other, the AcrySof IOL had a significantly higher absolute PR-PPR difference (and thus margin of error) than the FineVision lens at day 1 , week 1 , and month 1 . Of note, the margin of error with the AcrySof IOL showed a hyperopic shift that was the most pronounced on day 1. Given the fact that AcrySof showed significant postoperative IOL shifts and changes in refraction in the very early postoperative period, it may be wiser for eye surgeons to wait until week 1 to choose the IOL power. 
Limited research on the correlation between preoperative biometric variables and postoperative IOL longitudinal shift motivated us to analyze the relationship between such variables, such as ACD, LT, and AL [31], and postoperative IOL longitudinal shift, refraction change, and PR-PPR. In analyzing the in-the-bag IOLs, we found significant positive correlations between IOL shifts and changes in PR from day 1 to week 1 postoperatively in terms of preoperative ACD and AL. We also observed a significant negative correlation between IOL shifts from postoperative day 1 to week 1 .

Our results indicate that the larger the preoperative ACD and the longer the preoperative $\mathrm{AL}$, the more the IOLs shifted and the more the PR changed in the early postoperative period. In addition, the smaller the preoperative LT, the more the IOLs shifted, and the more the PR changed. Therefore, preoperative ACD, AL, and LT appear to be predictive factors for estimating the degree of IOL shift and changes in PR. These results indicate that these preoperative variables may be promising predictive factors. A larger preoperative $\mathrm{ACD}$, longer preoperative $\mathrm{AL}$, and smaller preoperative LT were all associated with a larger margin of error of postoperative refraction, but to a lesser degree than observed with IOL shift and PR changes.

There were some limitations to our study. First, the detailed mechanism of postoperative IOL longitudinal shift in the in-the-bag fixated IOLs SN60WF and FineVision was not investigated. In addition, the effect of IOL material, which may potentially play an important role, was not assessed. Future research will be needed to assess whether the differences can be explained, even in part, due to IOL material. Second, this study investigated only one type of capsulorhexis-fixation IOL. Different capsulorhexis-fixation IOLs with different materials and designs should be investigated in the future. Third, one of the main research objectives of this study was to compare postoperative IOL behaviors between in-the-bag and capsulorhexis fixations. However, since the three types of IOLs are associated with various factors that may affect their postoperative behavior, this study did not precisely investigate the difference between in-the-bag and capsulorhexis fixation. To precisely compare different IOL fixation methods, the same IOL should be used in different placements. Finally, only a single center was used for the recruitment of patients in this study, which may have affected the generalizability of our results. Further studies across multiple institutions are needed to validate our results.

\section{CONCLUSIONS}

When eye surgeons choose in-the-bag-fixation IOLs, it is vital that they understand the nature of potential postoperative refraction changes and the margin of postoperative refraction error. Preoperative ACD, LT, and AL may be useful factors for predicting IOL shift, change in $\mathrm{PR}$, and to a lesser extent the degree of margin of error in clinical practice. Familiarity with these points could also contribute to improved accuracy of PPR. Additionally, our results indicate that in the early postoperative stage, capsulorhexis-fixation is more stable than in-thebag IOLs in terms of postoperative IOL shifts and changes in postoperative refraction; it also showed the lowest margin of error of predicted postoperative refraction. Capsulorhexis fixation may be the most stable and promising IOL fixation method for improving the accuracy of PPR.

\section{ACKNOWLEDGEMENTS}

Funding. This research received no financial support, and the journal's Rapid Service Fee was funded by the authors.

Medical Writing, and Editorial Assistance. Editorial support, in the form of medical writing, assembling tables, and creating high-resolution images based on authors' detailed directions, collating author comments, copyediting, fact checking, and referencing, was provided by Editage, Cactus Communications. 
Authorship. All named authors meet the International Committee of Medical Journal Editors (ICMJE) criteria for authorship for this article, take responsibility for the integrity of the work as a whole, and have given their approval for this version to be published.

Authorship Contributions. Takeshi Teshigawara, Akira Meguro, and Nobuhisa Mizuki all contributed to the concept and design and the drafting and production of this manuscript.

Compliance with Ethics Guidelines. Informed consent was obtained from all patients. All research procedures were performed in accordance with the Declaration of Helsinki. This study was approved by the Ethics Committee of the Yokosuka Chuo Eye Clinic.

Disclosures. Takeshi Teshigawara, Akira Meguro, and Nobuhisa Mizuki declare no conflict of interest.

Data Availability. The datasets generated and/or analyzed during the current study are available from the corresponding author upon reasonable request.

Consent for Publication. Written informed consent was provided by all patients after a detailed explanation of this research.

Open Access. This article is licensed under a Creative Commons Attribution-NonCommercial 4.0 International License, which permits any non-commercial use, sharing, adaptation, distribution and reproduction in any medium or format, as long as you give appropriate credit to the original author(s) and the source, provide a link to the Creative Commons licence, and indicate if changes were made. The images or other third party material in this article are included in the article's Creative Commons licence, unless indicated otherwise in a credit line to the material. If material is not included in the article's Creative Commons licence and your intended use is not permitted by statutory regulation or exceeds the permitted use, you will need to obtain permission directly from the copyright holder. To view a copy of this licence, visit http://creativecommons.org/licenses/by$\mathrm{nc} / 4.0 /$.

\section{REFERENCES}

1. Gibbons A, Ali TK, Warren DP, Donaldson KE. Causes and correction of dissatisfaction after implantation of presbyopia-correcting intraocular lenses. Clin Ophthalmol. 2016;10:1965-70.

2. Olsen T. Sources of error in the intraocular lens power calculation. J Cataract Refract Surg. 1992;18: $125-9$.

3. Iqbal F, Khan HA. Comparative analysis of axial length measurement using partial coherence interferometry and clinical ultrasound. Adv Ophthalmol Vis Syst. 2019;9:11-3.

4. Melles RB, Holladay JT, Chang WJ. Accuracy of intraocular lens calculation formulas. Ophthalmology. 2018;125:169-78.

5. Nejima R, Miyai T, Kataoka $\mathrm{Y}$, et al. Prospective intrapatient comparison of 6.0-millimeter optic single-piece and 3-piece hydrophobic acrylic foldable intraocular lenses. Ophthalmology. 2006;113: 585-90.

6. Erickson P. Effects of intraocular lens position errors on postoperative refractive error. J Cataract Refract Surg. 1990;16:305-11.

7. Remón L, Siedlecki D, Cabeza-Gil I, Calvo B. Influence of material and haptic design on the mechanical stability of intraocular lenses by means of finite-element modeling. J Biomed Opt. 2018;23: $1-10$.

8. Ganesh B, Brar S. Swivel haptics anterior capsulefixated intraocular lens. Indian J Ophthalmol. 2018;66:1307-9.

9. Dick HB, Schultz T. Intraocular lens fixated in the anterior capsulotomy created in the line of sight by a femtosecond laser. J Refract Surg. 2014;30: 198-201.

10. Versace P, Smith DE. Femtis EDOF: Predictable Positioning, Clear Vision. mivision. 2019 Oct 28. Available from: https://www.mivision.com.au/ 2019/10/femtis-edof-predictable-positioning-clearvision/. Accessed 23 Feb 2020.

11. Dunn OJ. Multiple comparisons using rank sums. Technometrics. 1964;6:241-52. 
12. Khokhar S, Yadav D, Gupta S, et al. Refractive outcomes of cataract surgery in primary congenital glaucoma. Eye (Lond). 2019;33:542-8.

13. Mustafi D, Tom E, Messenger WB, Banitt MR, Rezaei KA. Outcomes of sutureless secondary intraocular lens fixation with haptic flanging in a cohort of surgically complex eyes. Graefes Arch Clin Exp Ophthalmol. 2020. https://doi.org/10.1007/ s00417-020-04937-9.

14. Wirtitsch MG, Findl O, Menapace R, et al. Effect of haptic design on change in axial lens position after cataract surgery. J Cataract Refract Surg. 2004;30: 45-51.

15. Kim KH, Kim WS. Intraocular lens stability and refractive outcomes after cataract surgery using primary posterior continuous curvilinear capsulorrhexis. Ophthalmology. 2010;117:2278-22786.

16. Gimbel HV, DeBroff BM. Intraocular lens optic capture. J Cataract Refract Surg. 2004;30:200-6.

17. Stodulka P, Packard R, Mordaunt D. Efficacy and safety of a new selective laser device to create anterior capsulotomies in cataract patients. J Cataract Refract Surg. 2019;45:601-7.

18. Chang DF. Zepto precision pulse capsulotomy: a new automated and disposable capsulotomy technology. Indian J Ophthalmol. 2017;65:1411-4.

19. Qian D-W, Guo H-K, Jin S-L, Zhang H-Y, Li Y-C. Femtosecond laser capsulotomy versus manual capsulotomy: a Meta-analysis. Int J Ophthalmol. 2016;9:453-8.

20. Kránitz K, Takacs A, Miháltz K, Kovács I, Knorz MC, Nagy ZZ. Femtosecond laser capsulotomy and manual continuous curvilinear capsulorrhexis parameters and their effects on intraocular lens centration. J Refract Surg. 2011;27:558-63.

21. Nagy ZZ, Takacs A, Filkorn T, Sarayba M. Initial clinical evaluation of an intraocular femtosecond laser in cataract surgery. J Refract Surg. 2009;25: 1053-60.

22. Eppig T, Scholz K, Löffler A, et al. Effect of decentration and tilt on the image quality of aspheric intraocular lens designs in a model eye. J Cataract Refract Surg. 2009;35:1091-100.

23. Iwase $T$, Tanaka N, Sugiyama K. Postoperative refraction changes in phacoemulsification cataract surgery with implantation of different types of intraocular lens. Eur J Ophthalmol. 2008;18:371-6.

24. Hayashi K, Hayashi H. Comparison of the stability of 1-piece and 3-piece acrylic intraocular lenses in the lens capsule. J Cataract Refract Surg. 2005;31: $337-42$.

25. Findl O, Drexler W, Menapace R, et al. Accurate determination of effective lens position and lenscapsule distance with 4 intraocular lenses. J Cataract Refract Surg. 1998;24:1094-8.

26. Koeppl C, Findl O, Kriechbaum K, Sacu S, Drexler W. Change in IOL position and capsular bag size with an angulated intraocular lens early after cataract surgery. J Cataract Refract Surg. 2005;31: 348-53.

27. Petternel V, Menapace R, Findl O, et al. Effect of optic edge design and haptic angulation on postoperative intraocular lens position change. J Cataract Refract Surg. 2004;30:52-7.

28. Akkin C, Ozler SA, Mentes J. Tilt and decentration of bag fixated intraocular lenses: a comparative study between capsulorhexis and envelope techniques. Doc Ophthalmol. 1994;87:199-209.

29. Kimura W, Kimura T, Sawada T, et al. Postoperative decentration of three-piece silicone intraocular lenses. J Cataract Refract Surg. 1996;22:1277-80.

30. Guthoff R, Abramo F, Draeger J, Chumbley LC, Lang GK, Neumann W. Forces on intraocular lens haptics induced by capsular fibrosis: an experimental study. Graefes Arch Clin Exp Ophthalmol. 1990;228:363-8.

31. Yoo SY, Whang JW, Kim SH. Preoperative biometric measurements with anterior segment optical coherence tomography and prediction of postoperative intraocular lens position. Medicine (Baltimore). 2019;98:e18026. 